\title{
Integration of IEEE C37.118 and Publish/Subscribe Communication
}

\author{
Michael Hoefling*, Florian Heimgaertner*, Daniel Fuchs*, Michael Menth*, \\ Paolo Romano ${ }^{\dagger}$, Teklemariam Tesfay ${ }^{\dagger}$, Mario Paolone ${ }^{\dagger}$, Jimmie Adolph ${ }^{\ddagger}$, and Vidar Gronas ${ }^{\ddagger}$ \\ *University of Tuebingen, Chair of Communication Networks, Tuebingen, Germany, Email: hoefling@uni-tuebingen.de \\ ${ }^{\dagger}$ Swiss Federal Institute of Technology in Lausanne (EPFL), Distributed Electrical System Laboratory, Lausanne, Switzerland \\ ${ }^{\ddagger}$ National Instruments, Stockholm, Sweden
}

\begin{abstract}
IEEE C37.118 is the current standard for synchrophasor measurements in power systems. It defines the measurement method and communication protocols for the entities in a synchrophasor network. The standard offers two different modes for client-server communication, but cannot be used unchanged over publish/subscribe communication architectures, whose major advantage is simplified and incremental integration of new applications. This work reviews the communication part of IEEE C37.118, and provides an adapter-based solution to easily connect and integrate entities in a synchrophasor network over a publish/subscribe communication architecture. The proposed adapters offer standard-compliant communication between the synchrophasor measurement network entities to facilitate the exchange of measurement data.
\end{abstract}

\section{INTRODUCTION}

Electrical power distribution networks are undergoing major changes in operational procedures and monitoring, thereby evolving from passive to active networks [1], [2]. Advanced smart monitoring tools result in faster and more reliable realtime state estimation (RTSE) [3]. Especially the massive use of phasor measurement units (PMUs) can achieve a more complete view and improve control of power networks [3], [4], [5]. IEEE C37.118 is the current standard for synchrophasor measurements in power systems, defining the measurement method [6] and communication protocols [7] for the entities in a synchrophasor network. It offers two different modes for client-server communication over TCP or UDP between PMUs and phasor data concentrators (PDCs), but cannot be used unchanged over publish/subscribe (pub/sub) communication architectures.

The main motivation for the introduction of pub/sub communication in smart grids is improved scalability with regard to the number of communication partners, and ease of application development [8], [9]. Publishers and subscribers are decoupled in the sense that they do not need to know each other a priori, rather a message broker ensures that messages are relayed between registered publishers and subscribers of a topic. In this context, a topic is an abstract representation of a unidirectional information channel, and is addressed using its unique name and probably attributes, e.g., data type, location, and time. An example for a topic is phasor measurement data for a specific geographic region inside the electrical power distribution network.

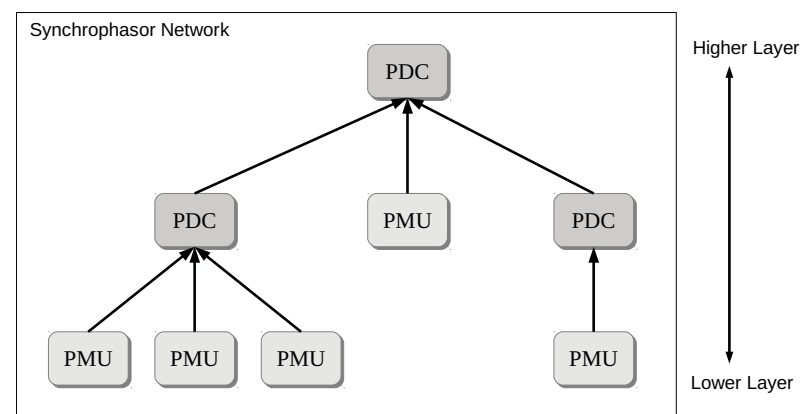

Fig. 1. An example of an IEEE C37.118 synchrophasor network.

The main contribution of this paper is an adapter-based method to connect and integrate IEEE C37.118 communication entities over a pub/sub communication architecture. Our proposed solution offers standard-compliant communication between the synchrophasor measurement network entities, i.e, existing IEEE C37.118-compliant hardware and software is able to communicate over a pub/sub system. The solution presented here is implemented in the pub/sub C-DAX [10] middleware prototype and will be deployed in a field trial, but may be applied to other pub/sub systems as well.

This work is structured as follows. We review relevant aspects of the IEEE C37.118 standard in Sect. II and briefly present the pub/sub communication paradigm in Sect. III. In Sect. IV, we explain how our proposed publisher and subscriber adapters work and interface with IEEE C37.118compliant devices and the pub/sub system. We discuss related work in Sect. V and draw conclusions in Sect. VI.

\section{IEEE C37.118: A STANDARD FOR SYNCHROPHASOR MEASUREMENT IN POWER Systems}

IEEE C37.118 is the current standard for synchrophasor measurement in power systems and divided into two documents: one document describing the phasor measurement in power grids [6], and one document describing the communication architecture [7]. In the following, we describe the components and the communication in synchrophasor networks according to that standard. 


\section{A. Synchrophasor Networks}

A synchrophasor network is a hierarchically organized network and consists of two components: phasor measurment units (PMUs) and phasor data concentrators (PDCs). PMUs measure synchrophasors along a feeder, time-stamp each measurement using a reliable time source, such as GPS, and send the time-stamped measurement data to the PDC. PDCs receive measurement data from PMUs, aggregate data from different PMUs based on the time-stamp, and optionally forward the aggregated data to superordinate PDCs or provide them to applications. Fig. 1 shows an example of a twolevel synchrophasor network, and illustrates that measurement data flows are unidirectional from lower-layer to higher-layer devices.

The standard defines the terms server and client as follows. A server is a function or device providing phasor measurement data, e.g., a PMU or an intermediate PDC. A client is a function or device receiving phasor measurement data, e.g., a PDC. An intermediate PDC may also provide measurement data to another PDC. As a result, a device can be both server and client. While the server-side function is providing measurement data to other devices, the client-side function is receiving measurement data. Each stream in a synchrophasor network device is identified and addressed by a 16-bit $I D$ CODE, i.e., IEEE C37.118 can be deployed on top of any transport protocol because information from lower protocol layers is ignored, e.g., IP address and port number.

\section{B. Messages}

The standard defines four types of messages: command (CMD), data (DATA), header (HDR), and configuration (CFG). CMD messages are sent from clients to servers and contain the server stream's IDCODE. They may switch data streaming on and off, or request CFG and HDR messages. DATA messages are sent from servers to clients, and contain the server stream's IDCODE, and single or aggregated PMU measurement data. They cannot be interpreted without knowing the current configuration of the PMU. Servers send client HDR messages containing general information about the PMU(s), scaling, algorithms, and filtering. HDR messages are not used for synchrophasor data streaming.

The standard defines three types of $C F G$ messages: CFG1, CFG-2, and CFG-3. CFG messages are sent from servers to clients and contain the server stream's IDCODE. CFG-1 messages contain generic capability information of the queried PMU device and are not used in the data streaming context. CFG-2 messages contain the current configuration of the PMU device which is necessary to interpret the measurement data. CFG-3 messages are extended CFG-2 message enabling advanced phasor measurement features, e.g., flexible framing or global PMU IDs. We will use CFG and CFG-2 for configuration messages interchangeably.

\section{Communication Modes}

The standard describes two modes of operation with regard to communication: commanded mode and spontaneous mode.

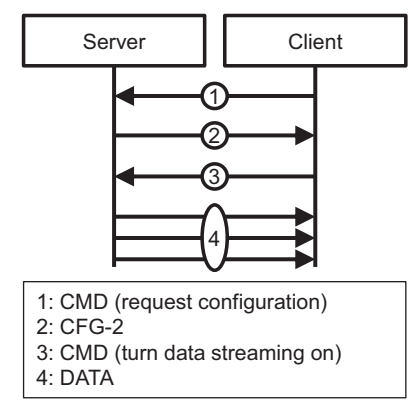

(a) Commanded mode. Servers send DATA and CFG messages to clients on request.

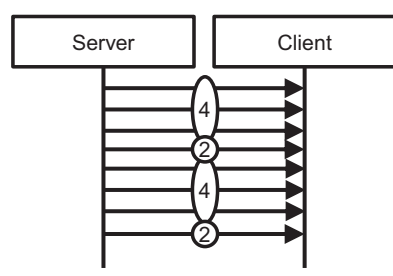

2: CFG-2

4: DATA

(b) Spontaneous mode. Servers send DATA and CFG messages to clients unsolicitedly.
Fig. 2. IEEE C37.118 communication modes. Servers are functions or devices providing phasor measurement data, e.g., PMUs or intermediate PDCs. Clients are functions or devices receiving phasor measurement data, e.g., PDCs.

1) Commanded Mode: PMUs and PDCs interact with each other using bidirectional communication in commanded mode, as shown in Fig. 2(a). First, the PDC requests the PMU configuration by sending a CMD message with the request$C F G-2$ option to the PMU. After successful retrieval of the CFG-2 message, the PDC switches data streaming on by sending a CMD message with the turn-streaming-on option to the PMU. Eventually, the PMU starts to continuously stream measurement data to the PDC. The PMU triggers its PDC to actively request a new CFG-2 message if the PMU configuration changes. The PDC sends a CMD message with the turn-streaming-off option to the PMU to switch data streaming off. The standard recommends using UDP for measurement data but supports TCP as well. Further, having a TCP-based control channel and a UDP-based data channel is also possible.

2) Spontaneous Mode: In contrast to commanded mode, PMUs send unsolicited DATA and CFG messages to PDCs over UDP in spontaneous mode, i.e., there is no communication in the reverse direction, as shown in Fig. 2(b). The rate of DATA messages depends on the PMU measurement configuration. The rate of $\mathrm{CFG}-2$ messages depends on the general PMU configuration. Streaming CFG-2 messages in regular intervals is necessary because PDCs can interpret the received measurement data only after receiving the current configuration of the PMUs, i.e, the PDC automatically learns about a PMU configuration change without any additional communication overhead.

\section{Publish/Subscribe BASICS}

The basic idea of the pub/sub paradigm is the decoupling of communication partners in space, time, and synchronization. The goal is to improve scalability compared to traditional client-server communication, and to facilitate development of new communication-based applications by providing a standardized transparent interface [8], [9]. We review components and signaling in typical pub/sub systems and give a brief introduction to C-DAX, the pub/sub system for which we developed the IEEE C37.118 adapter. 


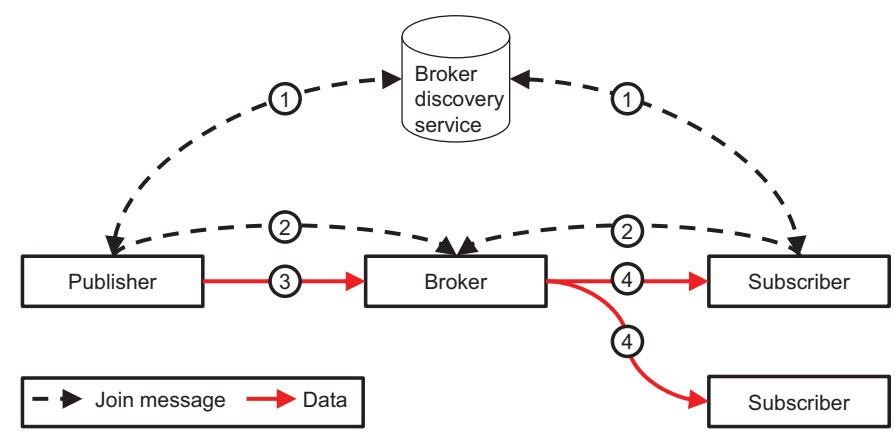

Fig. 3. Basic pub/sub communication. After successful broker discovery (step 1) and client join (step 2), publishers send data to brokers (step 3) which forward data to subscribers (step 4).

\section{A. Components and Signaling}

A pub/sub communication architecture consists of at least four components: publishers, subscribers, brokers, and a broker discovery service. Pub/sub communication can be topicoriented, content-based, or type-based [8]. We are interested in topic-oriented pub/sub communication only. First, publishers and subscribers register with a broker for a certain topic. Then, publishers send messages for that topic to that broker which forwards them to the subscribers. A broker discovery service tells publishers and subscribers what broker supports the communication on a certain topic. Depending on deployment, the functionality of brokers may be collocated with publishers or subscribers.

The signaling interactions of typical pub/sub architectures are illustrated in Fig. 3. In the step 1, publishers and subscribers query the broker discovery service to find the appropriate broker for further communication on a certain topic. In step 2, publishers and subscribers send a join message to the broker; the message also indicate the role of publishers and subscribers. After successful join, publishers may start sending data to the broker in step 3. In step 4, the broker eventually starts forwarding data to the registered subscribers in step 4.

\section{B. C-DAX: A Pub/Sub Middleware for Power Grids}

The Cyber-secure Data and Control Cloud for power grids (C-DAX) project [10] is an FP7 project funded by the European Commission which adapts the pub/sub paradigm to the needs of power grids. It aims to develop a cybersecure communication middleware for smart grids, applying the pub/sub paradigm to enable scalable, transparent, and secure end-to-end communication [11] between publishers and subscribers. Additional major advantages of the C-DAX architecture include resilient communication [12], inter-domain communication, and support for real-time applications [13].

Resilient data forwarding and data redundancy enable a high level of reliability, i.e., C-DAX provides reliable communication without service degradation should any component fail. C-DAX enables utilities to share information with other utilities in a secure and scalable manner, facilitating interdomain communication. A cyber-security layer provides authentication, privacy, and integrity in an end-to-end fashion. C-
DAX enables integration of emerging smart grid applications giving grid operators full flexibility. The data plane supports different communication modes including streaming, query, and point-to-point communication.

RTSE is a use case of C-DAX which will be demonstrated as part of the project's field trial in the Alliander LiveLab [14] smart grid test site. RTSE requires reliable and timely delivery of phasor measurement data from PMUs to PDCs. Therefore, the pub/sub C-DAX middleware needs to interface with PMUs and PDCs. Due to its flexibility, C-DAX can also support other smart grid applications such as substation automation or retail energy transactions. However, these use cases are only covered conceptually as part of the project.

\section{INTEGRATION OF IEEE C37.118 IN Publish/Subscribe COMMUNiCATION}

In this section, we clarify the need for integration of IEEE C37.118 in pub/sub communication. We propose the concept of publisher and subscriber adapters, and show how they can solve the problem. We discuss implementation alternatives, their pros and cons, and configuration considerations. Finally, we summarize the current implementation.

\section{A. The Need for Integration of IEEE C37.118 in Pub- lish/Subscribe Communication}

Existing products for PMUs and PDCs implement IEEE C37.118 as communication interface. Commanded mode is not suitable for pub/sub communication because it requires bidirectional one-to-one communication, and pub/sub systems support unidirectional many-to-many communication only. Spontaneous mode does not require a reverse channel, i.e., data delivery over pub/sub is meaningful but additional configuration on the PMU side is necessary to properly set a streaming target. Commanded mode is widely used in existing largescale PMU installations, e.g., the Synchrophasors Initiative in India [15]. Additionally, off-the-shelf products for PMUs and PDCs may offer only commanded mode prohibiting data transport over pub/sub communication infrastructures, e.g., the open-source iPDC [16] simulator suite. To include such PMUs or PDCs in settings with pub/sub communication, translations between commanded mode and spontaneous mode are needed before and after data are transmitted over pub/sub. Furthermore, IEEE C37.118 messages need to be translated into a format compatible with the pub/sub data plane and converted back when delivered to the application. Otherwise, the pub/sub communication architecture cannot process and forward IEEE C37.118 messages.

\section{B. Publisher and Subscriber Adapters}

We propose publisher and subscriber adapters that perform communication mode and message translation to integrate IEEE C37.118 with pub/sub communication. Adapters provide interfaces for native pub/sub and IEEE C37.118 communication. They are full-fledged publishers and subscribers, and allow clients and servers of a synchrophasor network to exchange data over a pub/sub communication infrastructure. 


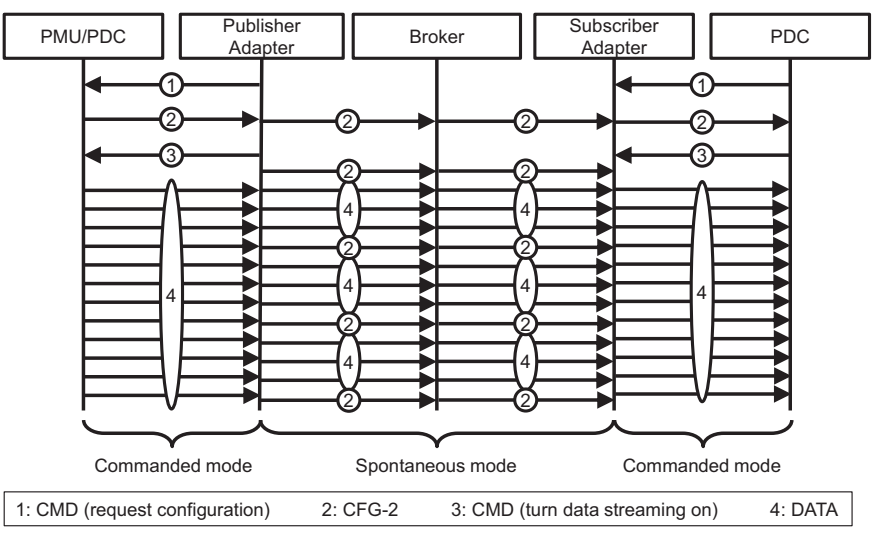

Fig. 4. Communication mode translation of publisher and subscriber adapters. The publisher adapter requests CFG-2 frames from the server, toggles data streaming, and forwards DATA frames with interspersed CFG-2 frames over the pub/sub communication infrastructure to the subscriber adapter. The subscriber adapter listens for incoming commands from the client and forwards CFG-2 and DATA frames on request.

When a publisher or subscriber adapter is started, it first joins the pub/sub network as a publisher or subscriber for its pre-configured topics. After appropriate signaling with a server (PMU or PDC), the publisher adapter sends synchrophasor data over the network. Likewise, the subscriber adapter forwards synchrophasor data to the client PDC after appropriate signaling.

All subscriber adapters receive the same topic data should they join the same topic, e.g., a second PDC is able to process the topic data because it receives the same CFG2 and DATA messages as the first PDC. Should no topic data be available for a topic because no publishers exists yet, the subscriber adapter signalizes that according to the IEEE C37.118 protocol. Subscriber adapters silently discard all received DATA messages if their PDC did not switch on data streaming; this is valid behavior because applications like RTSE rely on timely DATA messages.

\section{Communication Mode Translation}

We introduce two types of translation behaviors that enable an adapter to translate between spontaneous and commanded mode, depending on whether the adapter is located at server/publisher side or client/subscriber side. Communication mode translation is not necessary when PMUs and PDCs are both operated in spontaneous mode.

Fig. 4 illustrates the basic idea behind communication mode translation. In the first case, data come from a server (PMU or PDC) that uses commanded mode and is translated to spontaneous mode before being passed to a publisher to be carried over the pub/sub communication infrastructure (left side of Fig. 4). In the second case, data come from the pub/sub communication infrastructure via a subscriber in spontaneous mode and are translated to commanded mode before being passed to a client that uses commanded mode (right side of Fig. 4). Publisher adapters perform commandedto-spontaneous mode translation; subscriber adapters perform spontaneous-to-commanded mode translation. We explain the translation operations inside both adapters.

1) Publisher Adapter: A publisher adapter initiates data transmission with a server which may be a PMU or a sending PDC. It translates from commanded mode to spontaneous mode which is depicted in Fig. 4. The adapter communicates with the server (PMU) in commanded mode. It requests a CFG-2 message from the server and then stores it internally, so that the configuration remains available. The streaming from the server to the adapter is enabled by a CMD message. The adapter starts sending the CFG-2 message spontaneously over the pub/sub communication infrastructure via a subscriber to the client (PDC) in user-defined intervals. The adapter forwards the incoming DATA messages to the pub/sub communication infrastructure.

2) Subscriber Adapter: A subscriber adapter communicates with the client PDC in commanded mode, meaning that it listens for CMD messages from the client PDC, as depicted in Fig. 4. It receives CFG-2 and DATA messages spontaneously from the pub/sub communication infrastructure via the subscriber, and stores CFG-2 messages internally for future use. The client PDC requests a CFG-2 message from the adapter to correctly interpret the measurement data. Then, the client sends a CMD message to the adapter to turn on the data streaming, which is in fact a forwarding of DATA messages from the server. Finally, the client turns off the data streaming of the adapter in a similar manner. Without translation at the subscriber side, the receiving PDC is served exclusively and transparently in spontaneous mode.

\section{Message Translation}

We propose to use data decapsulation and re-encapsulation as a straightforward solution for translating IEEE C37.118 messages to pub/sub data plane messages. The message translation step may be simplified if the adapters are integrated on the PMU/PDC platform.

1) Publisher Adapter: A server (PMU or PDC) sends messages to a publisher adapter which strips off the TCP/UDP and IP headers to obtain the actual IEEE C37.118 messages. The publisher adapter re-encapsulates these messages in the data plane format of the underlying pub/sub architecture. Additionally, it may extract data fields from the original IEEE C37.118 messages and embed this information in the data plane message header, e.g., for in-network filtering if the pub/sub architecture supports this feature.

2) Subscriber Adapter: On the receiving side, a subscriber adapter translates the pub/sub data plane message back before handing it over to the PDC. This is done by decapsulating the original IEEE C37.118 message from the data plane message, encapsulating it in TCP/UDP and IP, and re-sending it towards the PDC. Additionally, the adapter performs stream demultiplexing towards the PDC if the PDC cannot separate distinct PMU streams received over the same TCP/UDP socket, e.g., by assigning a unique source port number to each PMU stream. 


\section{E. Implementation Options for Publisher and Subscriber Adapters}

The adapter can be implemented (1) as an additional software module of the target PMU/PDC platform, or (2) as an extra logical and physical entity. We briefly discuss the advantages and disadvantages of both approaches. The advantage of approach (1) is that adapters integrated into the PMU/PDC platform do not add transmission delay, but its disadvantage is increased integration effort as coding on the PMU/PDC platform is required. It may be even difficult or impossible to add code on these platforms so that only the second approach may be feasible. The advantage of approach (2) is that PMU and PDC platforms do not need to be modified. Their configuration just has to be pointed to the adapters which take care of the necessary signaling and data forwarding. Its disadvantage is that the adapters introduce extra hops on the communication path which adds delay, e.g., on the station bus. If PMU and PDC manufacturers take care of the integration of IEEE C37.118 in pub/sub, they should follow the first approach which is feasible for them and does not cause additional communication delay.

\section{F. Configuration Considerations}

The configuration of the synchrophasor network has a direct influence on the configuration of the adapters. In general, two cases have to be considered: the synchrophasor network is operated in spontaneous mode, and the synchrophasor network is operated in commanded mode. We assume that it is best practice to operate synchrophasor networks using the same communication mode for all deployed devices, i.e., we omit discussing cases with PMUs and PDCs using different communication modes inside the same synchrophasor network. Independent of the communication mode of the synchrophasor network, PMU adapters and PDC adapters always have to be configured with the correct topic names and the correct pub/sub credentials.

1) Synchrophasor Network Operated in Spontaneous Mode: PMUs have to be configured with the IP address and port number of the publisher adapter. The subscriber adapter has to be configured with the IP address and port number of the client PDC so that it can correctly forward data received over the pub/sub architecture towards that PDC. Publisher adapters and client PDCs do not need any further configuration.

2) Synchrophasor Network Operated in Commanded Mode: Client PDCs have to be configured with the IP address, port number, and the IDCODEs of all PMU streams of interest of the subscriber adapter. Subscriber adapters only have to be configured to translate between spontaneous and commanded mode, i.e., they automatically learn the IDCODEs of all PMU streams for which they are responsible for by processing the data received from the pub/sub communication infrastructure, and they react on IEEE C37.118 communication from the PDC. Publisher adapters have to be configured with the IP address, port number, and IDCODEs of their respective PMU streams, and they have to be configured to translate between commanded and spontaneous mode, i.e., they need to initiate communication with the PMUs. PMUs do not need any further configuration.

\section{G. Implementation in $C-D A X$}

We implemented the described publisher and subscriber adapters in the C-DAX prototype, and deployed our software on the Virtual Wall network testbed [17], [18]. Currently, the implementation supports spontaneous mode PMUs and PDCs over UDP only, performs stream demultiplexing at the subscriber adapter, and is realized as extra entities (see approach (2) in Sect. IV-E). We used our adapter implementation to connect four PMUs over the C-DAX communication architecture to one PDC. Additionally, we have a running proof-of-concept implementation of the communication mode translation, allowing to interconnect spontaneous and commanded mode PMUs and PDCs in any meaningful combination. The current implementation supports UDP as transport protocol only. We used the PMU Connection Tester [19] analysis software to verify the correctness of our implementation. The PMU Connection Tester supports IEEE C37.118 in commanded and spontaneous mode, and allows to investigate PMU communication. Further, we enhanced the iPDC [16] PDC/PMU simulator software with spontaneous mode to have a test target for our adapter, e.g., to generate spontaneous mode PMU data for testing and evaluating our adapter. We used the enhanced version of iPDC as a blueprint for our adapter during the early stage of our prototype development.

\section{RELATED WORK}

Eugster et al. [8] provide an excellent classification and overview on the pub/sub paradigm and existing architectures. Besides pub/sub, alternative communication paradigms are discussed as well. However, integration of application-specific protocols in pub/sub architectures is not covered.

SeDAX [9] and GridStat [20] are pub/sub architectures specifically targeting smart grids. They support the integration of different smart grid applications using a common communication API. However, neither of the architectures provide a description of what smart grid protocols are supported and how they are integrated.

The OMG Data-Distribution Service (DDS) [21] is a pub/sub architecture which targets real-time communication. Application-specific protocols are integrated utilizing the socalled data local reconstruction layer (DLRL). Application developers must define the communication interface using an interface definition language (IDL) to enable native communication over DDS.

NASPI (North American SynchroPhasor Initiative) operates a large-scale measurement infrastructure called NASPInet [22] which is composed of phasor gateways (PGs) and a data bus which is basically a DDS-based pub/sub architecture. PGs use the gateway exchange protocol (GEP) to translate and exchange phasor measurement data between applicationspecific PMU protocols and the internally used data bus system. Open-source implementations of PGs [23], [24], [25] are provided by the Grid Protection Alliance. 
IEC 61850 [26] is the industry standard for substation automation. It provides its pub/sub communication over the generic object oriented substation event (GOOSE) and the sampled values (SV) protocol. The extension IEC 61850-905 [27] describes mechanisms to map IEEE C37.118 to GOOSE and SV, thus enabling the use of IEC 61850 to transmit synchrophasor information conforming to the IEEE C37.118 standard. This facilitates PMU and PDC deployments being an integral part of substation planning, installation, and operation.

\section{CONCLUSIONS}

In this paper, we proposed a solution to easily connect and integrate entities in a IEEE C37.118 synchrophasor network over a pub/sub communication infrastructure. We introduced publisher and subscriber adapters as interfaces for entities of the synchrophasor network with the pub/sub architecture. The adapters translate between IEEE C37.118 commanded and spontaneous mode which is necessary as commanded mode requires a back channel that is unavailable in pub/sub communication. They also translate the message format so that the data can be forwarded over the pub/sub communication infrastructure. We explained these procedures in detail, discussed implementation options, and clarified configuration issues. Our proposed method allows transparent integration of all IEEE C37.118-compliant hardware and software in pub/sub architectures.

The adapter concept is already implemented in the C-DAX prototype as a standalone solution and will be used in the CDAX field trial. Future work includes closer integration of the adapter concept on existing PMU and PDC platforms, inclusion of communication mode translation in the running prototype, and the extension of our adapter to support other relevant smart grid protocols, e.g., the SCADA interconnection protocol IEC 60870-5-104 [28].

\section{ACKNOWLEDGMENT}

The research leading to these results has received funding from the European Community's Seventh Framework Programme FP7-ICT-2011-8 under grant agreement $\mathrm{n}^{\circ} 318708$ (C-DAX). The authors alone are responsible for the content of this paper.

The authors thank M. Thottan, K. V. Katsaros, C. Develder, C. Mills, and M. Schmidt for valuable input and stimulating discussions.

\section{REFERENCES}

[1] CIGRE Working Group C6.11, "Development and Operation of Active Distribution Networks," Apr. 2011.

[2] G. T. Heydt, "The Next Generation of Power Distribution Systems," IEEE Transactions on Smart Grid, vol. 1, no. 3, 2010.

[3] J. Liu, J. Tang, F. Ponci, A. Monti, C. Muscas, and P. A. Pegoraro, "Trade-Offs in PMU Deployment for State Estimation in Active Distribution Grids," IEEE Transactions on Smart Grid, vol. 3, no. 2, 2012.

[4] S. Sarri, M. Paolone, R. Cherkaoui, A. Borghetti, F. Napolitano, and C. Nucci, "State Estimation of Active Distribution Networks: Comparison between WLS and Iterated Kalman-Filter Algorithm Integrating PMUs," in IEEE PES International Conference and Exhibition on Innovative Smart Grid Technologies (ISGT Europe). IEEE, 2012.
[5] A. Borghetti, C. A. Nucci, M. Paolone, G. Ciappi, and A. Solari, "Synchronized Phasors Monitoring During the Islanding Maneuver of an Active Distribution Network," IEEE Transactions on Smart Grid, vol. 2, no. $1,2011$.

[6] "IEEE Standard for Synchrophasor Measurements for Power Systems," IEEE C37.118.1-2011, Dec. 2011.

[7] "IEEE Standard for Synchrophasor Data Transfer for Power Systems," IEEE C37.118.2-2011, Dec. 2011.

[8] P. T. Eugster, P. A. Felber, R. Guerraoui, and A.-M. Kermarrec, "The Many Faces of Publish/Subscribe," ACM Computing Surveys, vol. 35, no. 2, Jun. 2003.

[9] Y.-J. Kim, J. Lee, G. Atkinson, H. Kim, and M. Thottan, "SeDAX: A Scalable, Resilient, and Secure Platform for Smart Grid Communications," IEEE JSAC, vol. 30, no. 6, 2012.

[10] C-DAX Consortium, "Cyber-secure Data And Control Cloud for Power Grids," 2014. [Online]. Available: http://www.cdax.eu/

[11] B. Vieira and E. Poll, "A Security Protocol for Information-centric Networking in Smart Grids," in ACM Workshop on Smart Energy Grid Security (SEGS), Nov. 2013.

[12] M. Hoefling, F. Heimgaertner, M. Menth, K. V. Katsaros, P. Romano, L. Zanni, and G. Kamel, "Enabling Resilient Smart Grid Communication over the Information-Centric C-DAX Middleware," in ITG/GI International Conference on Networked Systems (NetSys), Cottbus, Germany, Mar. 2015.

[13] W. K. Chai, N. Wang, K. V. Katsaros, G. Kamel, S. Melis, M. Hoefling, B. Vieira, P. Romano, S. Sarri, T. Tesfay, B. Yang, F. Heimgaertner, M. Pignati, M. Paolone, M. Menth, G. Pavlou, E. Poll, M. Mampaey, H. Bontius, and C. Develder, "An Information-Centric Communication Infrastructure for Real-Time State Estimation of Active Distribution Networks," IEEE Transactions on Smart Grid, to appear.

[14] Alliander N.V., "LiveLab," 2015. [Online]. Available: https://www.alliander.com/en/innovation/our-innovations

[15] Power System Operation Corporation Limited, "Synchrophasors Initiative in India," Power System Operation Corporation Limited, Power Grid Corporation of India Limited, New Delhi, India, technical report, Dec. 2013.

[16] N. Pandit and K. Khandeparkar, "Design and Implementation of IEEE C37.118 based Phasor Data Concentrator \& PMU Simulator for Wide Area Measurement System," Indian Institute of Technology, Bombay, Tech. Rep., 2012.

[17] iMinds, "Virtual Wall - Generic test environment for advanced network, distributed software and service evaluation, and scalability research," 2014. [Online]. Available: http://www.iminds.be/en/develop-test/ilabt/virtual-wall

[18] S. Bouckaert, P. Becue, B. Vermeulen, B. Jooris, I. Moerman, and P. Demeester, "Federating Wired and Wireless Test Facilities through Emulab and OMF: The iLab.t Use Case," in International ICST Conference on Testbeds and Research Infrastructures for the Development of Networks and Communities. Ghent University, Department of Information Technology, 2012.

[19] Grid Protection Alliance, "PMU Connection Tester." [Online]. Available: http://pmuconnectiontester.codeplex.com/

[20] H. Gjermundrod, D. E. Bakken, C. H. Hauser, and A. Bose, "GridStat: A Flexible QoS-Managed Data Dissemination Framework for the Power Grid," IEEE Transactions on Power Delivery, vol. 24, no. 1, 2009.

[21] G. Pardo-Castellote, "OMG Data-Distribution Service: Architectural Overview," in ICDCS Workshops, 2003.

[22] P. T. Myrda and K. Koellner, "NASPInet - The Internet for Synchrophasors," in International Conference on Systems Sciences (HICSS). IEEE, 2010.

[23] Grid Protection Alliance, "SIEGate - the Secure Information Exchange Gateway," 2014. [Online]. Available: http://siegate.codeplex.com/

[24] - "openPG - the Open Source Phasor Gateway," 2013. [Online]. Available: http://openpg.codeplex.com/

[25] — " "substationSBG - the Substation Secure Buffered Gateway," 2014. [Online]. Available: http://substationsbg.codeplex.com/

[26] IEC, "IEC 61850: Power Utility Automation," 2003.

[27] —, "IEC 61850-90-5 ed1.0: Communication networks and systems in substations - Part 90-5: Use of IEC 61850 to transmit synchrophasor information according to IEEE C37.118," 2012.

[28] —, "IEC 60870-5-104 (IEC 104): Network access for IEC 60870-5101 using standard transport profiles," 2000. 